\title{
Informal Logic referees 2011-2012
}

The Editors express their gratitude and appreciation to the individuals listed below who served as referees for Informal Logic for Volumes 31 (2011) and 32 (2012).

Andrew Aberdein

Jonathan Adler $\dagger$

Scott Aiken

Mark Battersby

Lilian Bermejo-Luque

Jerome Bickenbach

John Biro

Charles Blatz

George Boger

Daniel Boone

Linda Carozza

Lorraine Code

Daniel Cohen

Michael Gary Duncan

Ian Dove

Daniel Fasko

Alec Fisher

Maurice Finochiaro

James Freeman

Mihai Frumuselu

Michael Gilbert

David Godden

Geoff Goddu

Jean Goodwin

Kara Gilbert

Marcello Guarini

Charles Hill

David Hitchcock
Michael Hoppmann

Catherine Hundleby

Ralph Johnson

Fred Kauffeld

Jerome Keppens

Harm Kloosterhuis

Tone Kvernbekk

Peter Loptson

Christoph Lumer

Sally Jackson

Fabrizio Macagno

Jim Mackenzie

Michael Malone

Hugo Mercier

Steven Patterson

Robert Pinto

Gilbert Plumer

William Rehg

Peter Schulz

Harvey Siegel

Dale Turner

Bart Verheij

MarkVorobej

Jean Wagemans

Douglas Walton

Larry Wright

Harald Wohlrapp

John Woods

(C) Informal Logic Editors. Informal Logic, Vol. 33, No. 1 (2013), p. 8o. 